\title{
Zeros de Polinômios Palindrômicos de Grau Ímpar
}

\author{
Junior A. Pereira Vanessa Botta \\ Depto de de Matemática e Computação, FCT, UNESP, \\ 19060-900, Presidente Prudente, SP \\ E-mail: junior.gusto@hotmail.com, botta@fctunesp.br
}

\section{RESUMO}

Seja $P(z)=a_{0}+a_{1} z+\ldots+a_{n} z^{n}$ um polinômio de grau $n, n \geq 1, a_{i} \in \mathbb{R}, i=0, \ldots, n$. Então $P$ é palindrômico se $a_{i}=a_{n-i}$, para todo $i=0,1, \ldots, n$. O comportamento dos zeros dos polinômios palindrômicos, além de ser um interessante tópico a ser estudado, tem muitas aplicações em algumas áreas da Matemática [2,6]. Tais zeros possuem algumas propriedades especiais, como a simetria tanto em relação à reta real quanto ao círculo unitário, por exemplo.

Atualmente, um tema que está atraindo a atenção de alguns matemáticos está relacionado à quantidade de zeros que um polinômio palindrômico possui no círculo unitário. Pesquisas recentes (ver $[3,4])$ estabelecem condições para que todos os zeros de um polinômio palindrômico estejam localizados em $|z|=1$.

Com base nos estudos de [3], apresentaremos, neste trabalho, condições necessárias e suficientes para que os zeros do polinômio palindrômico $R(z)=1+\lambda\left(z+z^{2}+\ldots+z^{n-1}\right)+z^{n}$, com $\lambda \in \mathbb{R}$ e $n$ ímpar, estejam localizados no círculo unitário, ou seja, na região $|z|=1$. Tais condições estão representadas no teorema abaixo, que será o foco principal deste trabalho, onde maiores detalhes podem ser encontrados em [1].

Teorema 1: Os zeros do polinômio $R(z)=1+\lambda\left(z+z^{2}+\ldots+z^{n-1}\right)+z^{n}, \lambda \in \mathbb{R}$, de grau $n>1$ ímpar, estão sobre o círculo unitário se, e somente se, $-\frac{2}{n-1} \leq \lambda \leq 2+\frac{2}{n-1}$.

Para exemplificar, seja o polinômio $R(z)=1+\lambda\left(z+z^{2}+z^{3}+z^{4}\right)+z^{5}$. Através do Teorema 1 , segue que os zeros de $R(z)$ encontram-se em $|z|=1$ se, e somente se, $-\frac{1}{2} \leq \lambda \leq \frac{5}{2}$. A Figura 1 mostra a localização dos zeros de $R(z)$ quando $\lambda=\frac{9.5}{4}$. Podemos observar que os zeros de $R(z)$ encontram-se em $|z|=1$, pois para $\lambda=\frac{9.5}{4}$ é satisfeita a condição do teorema. Já no caso da Figura 2, podemos observar que nem todos os zeros de $R(z)=1+3\left(z+z^{2}+z^{3}+z^{4}\right)+z^{5}$ encontram-se em $|z|=1$, pois a condição estabelecida através do Teorema 1 não é satisfeita. 


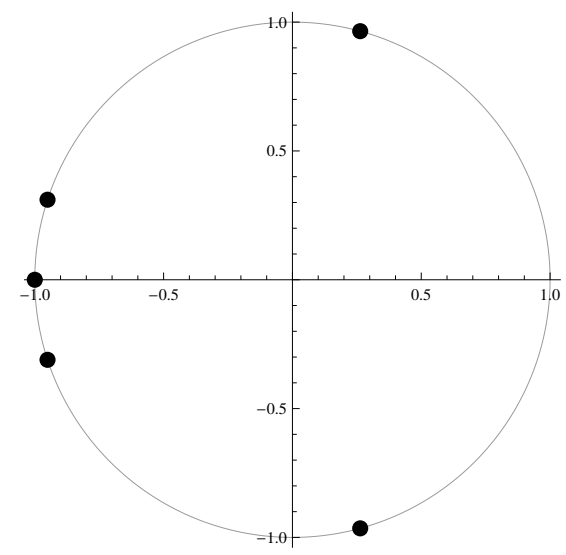

Figura 1: Zeros de

$R(z)=1+\frac{9.5}{4}\left(z+z^{2}+z^{3}+z^{4}\right)+z^{5}$.

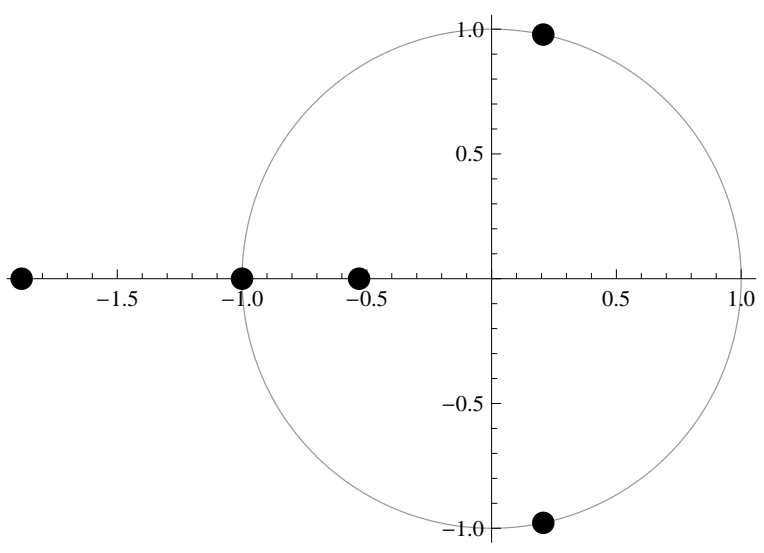

Figura 2: Zeros de

$R(z)=1+3\left(z+z^{2}+z^{3}+z^{4}\right)+z^{5}$.

Palavras-chave: Polinômio Palindrômico, Zeros de Polinômio, Círculo Unitário.

\section{Referências}

[1] V. Botta, L. F. Marques, M. Meneguete, Palindromic and Perturbed polynomials: zeros location. Acta Math. Hungar., 2013.

[2] L. Gemignani, V. Noferini, Modifications of Newton's method for even-grade palindromic polynomials and other twined polynomials, Num. Algorithms, 61 (2) (2012), pp. 315-329.

[3] D. Kwon, Reciprocal polynomials with all but two zeros on the unit circle, Acta Math. Hung., 134 (4) (2011), pp. 472-480.

[4] P. Lakatos, L. Losonczi, Polynomials with all zeros on the unit circle, Acta Math. Hung., 125 (4) (2009), pp. 341-356.

[5] G. V. Milovanóvic, D. S. Mitrinovic, Th. M. Rassias, Topics in Polynomials: Extremal Problems, Inequalities, Zeros, World Scientific, Singapore, 1994.

[6] M. R. Murty, C. Smyth, R. J. Wang, Zeros of Ramanujan Polynomials, J. Ramanujan Math. Soc., 26 (1) (2011), pp. 107-125. 